\title{
The Modeling and Analysis for the Self-Excited Vibration of the Maglev Vehicle-Bridge Interaction System
}

\author{
Jinhui Li, Jie Li, Danfeng Zhou, and Lianchun Wang \\ College of Mechatronics Engineering and Automation, National University of Defense Technology, Changsha 410073, China \\ Correspondence should be addressed to Jie Li; jieli@nudt.edu.cn
}

Received 27 June 2014; Accepted 1 March 2015

Academic Editor: Miguel A. F. Sanjuan

Copyright (C) 2015 Jinhui Li et al. This is an open access article distributed under the Creative Commons Attribution License, which permits unrestricted use, distribution, and reproduction in any medium, provided the original work is properly cited.

\begin{abstract}
This paper addresses the self-excited vibration problems of maglev vehicle-bridge interaction system which greatly degrades the stability of the levitation control, decreases the ride comfort, and restricts the cost of the whole system. Firstly, two levitation models with different complexity are developed, and the comparison of the energy curves associated with the two models is carried out. We conclude that the interaction model with a single levitation control unit is sufficient for the study of the self-excited vibration. Then, the principle underlying the self-excited vibration is explored from the standpoint of work acting on the bridge done by the levitation system. Furthermore, the influences of the parameters, including the modal frequency and modal damping of bridge, the gain of the controller, the sprung mass, and the unsprung mass, on the stability of the interaction system are carried out. The study provides a theoretical guidance for solving the self-excited vibration problems of the vehicle-bridge interaction systems.
\end{abstract}

\section{Introduction}

Compared with the conventional rail-way systems, the electromagnetic maglev system (EMS) has advantages of lower noise, less exhaust fumes emission, less maintenance cost, and the ability to climb steeper slopes, which is a new kind of urban transport that has been widely concerned in recent years [1-3].

The rapid development and enormous advantages of maglev system sketch a bright future for its commercial applications. However, the self-excited vibration of bridge is a burning issue to be solved. It occurs when the vehicle is suspended upon the guideway, standing still or moving at very slow speed [4]. It degrades the safety of bridge and durability of bridge. Furthermore, the self-excited vibration deteriorates the stability of the levitation system. The American maglev technologies (AMT) system achieved successful levitation in Florida on a bridge mounted to the earth on a concrete foundation but later encountered difficulties in achieving stable levitation when the vehicle was moved to an elevated bridge installed on the Old Dominion University campus [5]. It was believed that the flexibility of the bridge, which employed 90-feet long, was the main reason that contributed to the difficulties of achieving a stable levitation.

For the maglev CMS04 system of China, the self-excited vibration may occur if the parameters of PD controller are unsuitable. Figure 1 shows the recorded data from Tangshan maglev engineering base when the self-oscillation occurs.

According to Figure 1, when the self-excited vibration occurs, the electromagnet will vibrate vertically. Furthermore, the vibrations of electromagnet will be transferred to the cabin of vehicle, which is harmful for the riding comfort for passengers. Besides, the current fluctuations of the electromagnet impact the power system violently, which may lead to its collapse.

To explore the principle underlying the self-excited vibration and solve it, extensive researches have been reported. The derivation of bifurcation equations and numerical simulation using the center manifold method are carried out [6]. They believed that the bifurcations, such as the homoclinic, Hopf bifurcation, secondary Hopf bifurcation, and chaos are the causes of self-excited vibration. In [7] the authors believed that the self-excited vibration is due to the improper frequency relationship between various 

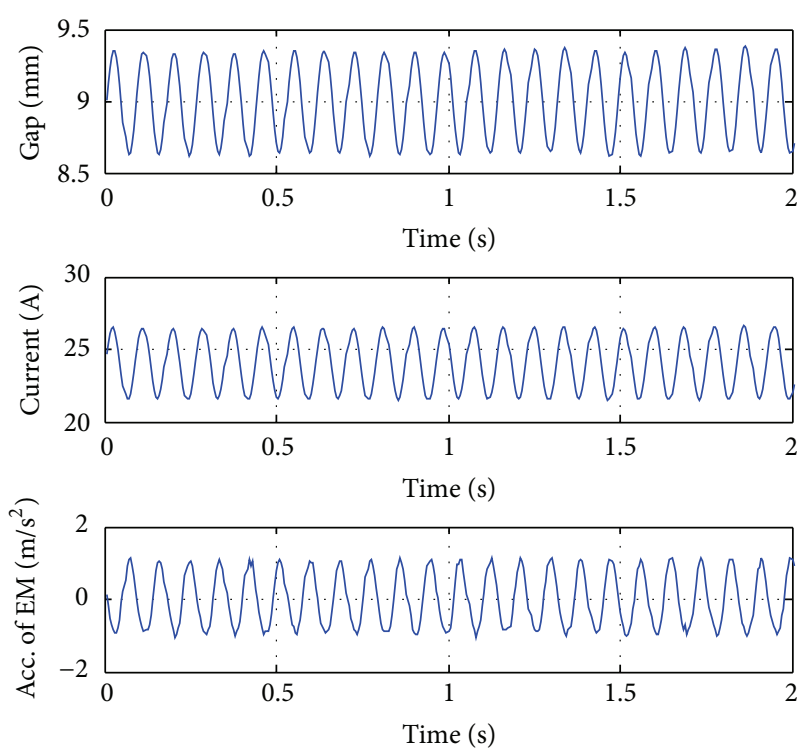

Figure 1: Recorded data from field when the self-oscillation occurred.

components of the system. In [8] the authors believed that the self-excited vibration is more likely to occur if the difference between the modal frequency of bridge and the natural frequency of controller is sufficiently small. The influences of signal delay on the stability of nonlinear levitation system are studied by [9-11]. The analysis shows that when the time delay reaches a critical value, the system will undergo a subcritical bifurcation, and the periodic vibration will occur.

The bridge in the maglev route is simply supported, and its span is large compared with other dimensions, and the vertical deflection of the bridges is small compared with its span when the self-excited vibration occurs.

In almost all the published literatures [12, 13], the elevated bridge is modeled as a Bernoulli-Euler beam. A consensus in maglev field has been reached, which will be adopted in this paper.

However, the development of the levitation model is relatively complicated. There have been many magnetic levitation models created for different purposes. Generally, these models are classified into two categories, one for the research of dynamics [14] and the other for the analysis and synthesis of the control system [15].

For the first kind, to obtain a precise and creditable dynamic response, the details of the system should be considered in all directions, so that this kind of model is relatively complicated. For the second kind, to simplify the process of analysis and synthesis of the control system, some inessential parts should be neglected and only the quintessential parts should be included.

For the analysis and synthesis of the self-excited vibration, various models with different complexities are adopted in published literatures. In [2], it is believed that the secondary suspension system of the vehicle can be neglected in the analysis, and the model of a single levitation unit-bridge coupled system was adopted. However, in [16], the structure of a single levitation unit-bridge with secondary suspension system was included. In [17], a complicated interaction model with a levitation module and secondary suspension system are developed.

Yet as of today, to the authors' knowledge, no effective theoretical method has been reported for the selection of a suitable minimum interaction model. As we all known, the rather larger error may be introduced if an oversimplified levitation model is selected. On the contrary, the theoretical derivation may be hard to be carried out if a complicated levitation model is adopted.

Therefore, a minimum interaction model with essential parts for the exploration of the principle underlying the self-excited vibration should be determined. In this paper, an index $Q$ that reflects the essence of the self-excited vibration will be defined. The feasibility of the index is analyzed. Furthermore, a minimum interaction model will be determined according to the comparison of the index $Q$.

Furthermore, based on the developed minimum interaction model, the principle underlying the self-excited vibration will be explored from the standpoint of work acting on the bridge done by the levitation system, and the frequency range of the vibration will be analyzed if the self-excited vibration occurs.

It has been observed that the PD controller cannot stabilize the vehicle-bridge interaction system completely when the vehicle is suspended on different bridges still. To solve the problem of self-excited vibration, the method to tune the parameters of controller and design the parameters of the bridges will be studied in this paper. To study the effects of varying parameters on closed-loop pole locations, the root locus method is adopted in this paper. In turn, these locations provide important information on the time and frequency responses, which are useful for the design guidance for the maglev system.

This study is engineering oriented, and the purpose of this research is to develop a minimum model that is capable of exploring the principle underlying the self-excited vibration and mastering the influences of each parameter on the stability of the interaction system.

\section{The Modeling of Vehicle-Bridge Interaction System}

Considering the complexity of self-excited vibration, the overall dynamic models with details may result in a difficult analysis to draw useful conclusions. Hence, a minimum interaction model containing quintessential parts associated with the occurrence of self-excited vibration should be developed.

2.1. The Modeling of Levitation System. The side view of the urban maglev vehicle, CMS04, is shown in Figure 2. It consists of ten levitation modules, which are designed to enable transmission of vertical force to support the vehicle body, to transmit lateral force to guide the train, and to transmit longitudinal forces for braking and traction force. This is to say, the levitation module is the minimum construction and dynamical unit. 


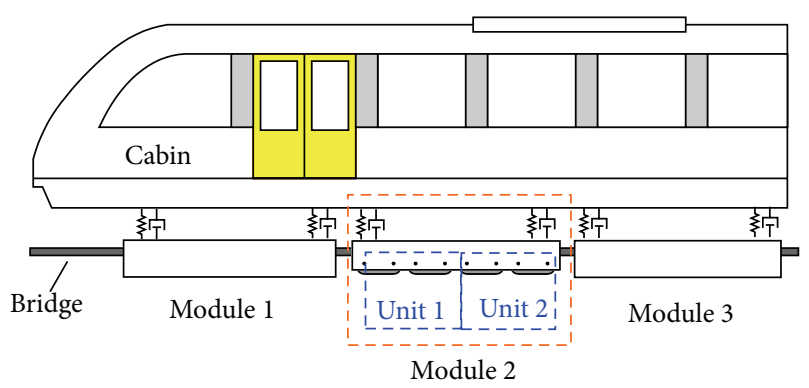

FIgURE 2: The side views of the CMS04 maglev system.

The module consists of four electromagnets. Each pair of adjacent electromagnets is controlled by a controller and is appointed as a levitation unit in this paper. This is to say, there are two levitation units for a levitation module.

Strictly speaking, the ten levitation modules of vehicle are lightly coupled by the movement of the cabin. However, if the overall maglev vehicle system is selected as a subject of model for the principle underlying the self-excited vibration, the model may be too complicated to carry out the theoretical investigation.

Generally, the influences of the interaction between the ten levitation modules on the stability of the vehiclebridge interaction system are neglected. Therefore, the overall system may be divided into ten independent subsystems, and each subsystem consists of a module, air-springs, and equivalent cabin, which are shown in Figure 3.

If the above subsystem is selected as a subject of the levitation model, the dynamic coupling between the two adjacent levitation units, the dynamics behavior of the air-springs, and the noncollocation between the sensors and actuators will be considered in all directions. Hence, the conclusions based on this subsystem will be creditable. Furthermore, if the above factors do not significantly affect the essence of the selfexcited vibration of the vehicle-bridge interaction system, then a simpler model, shown in Figure 4, may be preferable for the explanation of the self-excited vibration.

To explain the feasibility of the interaction model with a single levitation unit, an index $Q$ that reflects the essence of the self-excited vibration should be defined. Generally, the index $Q$ should possess a clear physical meaning to guide the optimization design and a brief format to carry out the theoretical derivation easily.

It has been observed that the bridge vibrates vertically when the self-excited vibration occurs. With the passage of time, the amplitude of vibration increases until the failure of the levitation system or self-oscillation with constant amplitude.

Generally, the growth of the vibration amplitude is attributed to the work done by the electromagnetic force acting on the bridge. Evidently, if the separation angle between the vertical velocity of the bridge $\dot{y}_{B}(t)$ and the electromagnetic force $F_{E}(t)$ acting on the bridge is less than $0.5 \pi$, the levitation system will export energy to the bridge, leading to the amplitude growth of the vibration. On the contrary, the amplitude of the vibration will decay to zero.

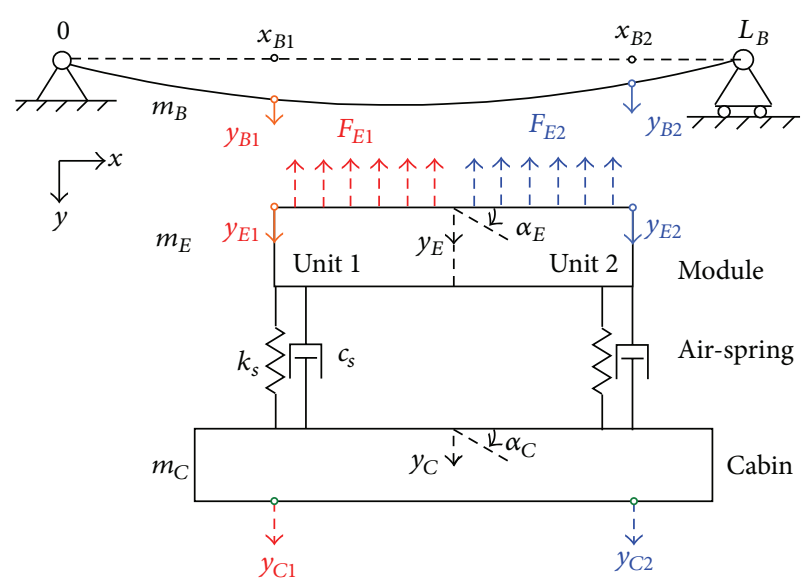

FIGURE 3: The interaction model with a module, the air-springs, and cabin.

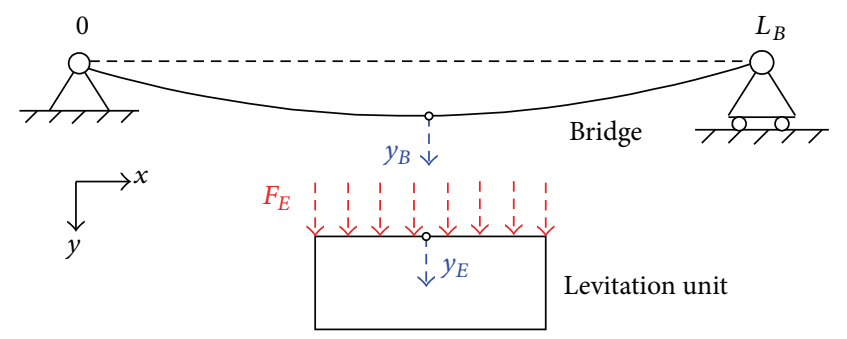

FIGURE 4: The interaction model with a single levitation unit.

Therefore, the transfer function between $\dot{y}_{B}(t)$ and $F_{E}(t)$ may be available for the definition of the index $Q$ to carry out the comparison of the two levitation models, shown in Figures 3 and 4 .

Firstly, the corresponding models should be developed. Considering the similarity of the two models and the limited length of the paper, only the dynamic equations of the interaction system with a module, the air-springs, and cabin are listed.

In light of Figure 4, the vertical displacements of the bridge are

$$
\begin{aligned}
& y_{B 1}=q_{1} \sin \left(\lambda_{1} x_{B 1}\right), \\
& y_{B 2}=q_{1} \sin \left(\lambda_{1} x_{B 2}\right) .
\end{aligned}
$$

The levitation gaps measured by the gap sensor are

$$
\begin{aligned}
& \delta_{1}=y_{E 1}-y_{B 1}, \\
& \delta_{2}=y_{E 2}-y_{B 2} .
\end{aligned}
$$

However, for the dynamic of the levitation system, its electromagnetic forces are associated with the averaged electric clearances $\bar{\delta}_{i}$ between the upper surface of the electromagnets and the lower surface of the guideway, which can be given as

$$
\begin{aligned}
& \bar{\delta}_{1}=\left(0.75 y_{E 1}+0.25 y_{E 2}\right)-q_{1} \sin \left(\lambda_{1}\left(x_{B 1}+L_{E}\right)\right), \\
& \bar{\delta}_{2}=\left(0.25 y_{E 1}+0.75 y_{E 2}\right)-q_{1} \sin \left(\lambda_{1}\left(x_{B 2}-L_{E}\right)\right) .
\end{aligned}
$$


Suppose that the number of turns of a single electromagnet is $N$, the pole area is $A$, and the magnetic permeability of vacuum is $\mu_{0}$. Then, for a single electromagnet, the relationship between the controlled voltage $u_{i}(t)$ and current $i_{i}(t)$ is

$$
\begin{aligned}
& \frac{u_{1}(t)}{2}=i_{1}(t) R+\frac{\mu_{0} A N^{2}}{2 \bar{\delta}_{1}(t)} \dot{i}_{1}(t)-\frac{\mu_{0} A N^{2} i_{1}(t)}{2 \bar{\delta}_{1}^{2}(t)} \dot{\bar{\delta}}_{1}(t), \\
& \frac{u_{2}(t)}{2}=i_{2}(t) R+\frac{\mu_{0} A N^{2}}{2 \bar{\delta}_{2}(t)} \dot{i}_{2}(t)-\frac{\mu_{0} A N^{2} i_{2}(t)}{2 \bar{\delta}_{2}^{2}(t)} \dot{\bar{\delta}}_{2}(t) .
\end{aligned}
$$

Here, $R$ is the resistance of a single electromagnet. The electromagnetic forces acting on the bridge are

$$
\begin{aligned}
& F_{E 1}(t)=\frac{\mu_{0} A N^{2}}{2}\left(\frac{i_{1}(t)}{\bar{\delta}_{1}(t)}\right)^{2}, \\
& F_{E 2}(t)=\frac{\mu_{0} A N^{2}}{2}\left(\frac{i_{2}(t)}{\bar{\delta}_{2}(t)}\right)^{2} .
\end{aligned}
$$

When the vehicle is suspended on the bridge without moving, the forces acting on the module include the gravity, the pressures from the distortion of the air springs, and the electromagnetic forces. The dynamic equations of first levitation modal are given as

$$
\begin{aligned}
J_{E} L_{E}^{-1} \ddot{\alpha}_{E}(t)= & 2 k_{s}\left(y_{E 1}-y_{C 1}-\Delta_{C E 0}\right)+2 c_{s}\left(\dot{y}_{E 1}-\dot{y}_{C 1}\right) \\
& +F_{E 1}(t)-F_{E 2}(t)-2 k_{s}\left(y_{E 2}-y_{C 2}-\Delta_{C E 0}\right) \\
& -2 c_{s}\left(\dot{y}_{E 2}-\dot{y}_{C 2}\right) \\
m_{E} \ddot{y}_{E}(t)= & -F_{E 1}(t)-k_{s}\left(y_{E 1}-y_{C 1}-\Delta_{C E 0}\right) \\
& -c_{s}\left(\dot{y}_{E 1}-\dot{y}_{C 1}\right)+m_{E} g-F_{E 2}(t) \\
& -k_{s}\left(y_{E 2}-y_{C 2}-\Delta_{C E 0}\right)-c_{s}\left(\dot{y}_{E 2}-\dot{y}_{C 2}\right) .
\end{aligned}
$$

The forces acting on the cabin of vehicle include the gravity and the counteracting force from the distortion of the air springs. The dynamic equations of the cabin are given as

$$
\begin{aligned}
m_{C} \ddot{y}_{C}(t)= & k_{s}\left(y_{E 1}-y_{C 1}-\Delta_{C E 0}\right)+c_{s}\left(\dot{y}_{E 1}-\dot{y}_{C 1}\right) \\
& +m_{C} g+k_{s}\left(y_{E 2}-y_{C 2}-\Delta_{C E 0}\right) \\
& +c_{s}\left(\dot{y}_{E 2}-\dot{y}_{C 2}\right), \\
J_{C} L_{C}^{-1} \ddot{\alpha}_{C}(t)= & -k_{s}\left(y_{E 1}-y_{C 1}-\Delta_{C E 0}\right)-c_{s}\left(\dot{y}_{E 1}-\dot{y}_{C 1}\right) \\
& +k_{s}\left(y_{E 2}-y_{C 2}-\Delta_{C E 0}\right)+c_{s}\left(\dot{y}_{E 2}-\dot{y}_{C 2}\right) .
\end{aligned}
$$

According to (6) and (8), the steady current of the electromagnet is

$$
i_{0}=\frac{\delta_{0}}{N} \sqrt{\frac{\left(m_{E}+m_{C}\right) g}{\mu_{0} A}} .
$$

Due to its clear physical meaning, the excellent performance, and easily engineering implementation, the integraltype PD control is the most widely used controller in engineering practice, which is preferred in this study. The integral-type PD controller is given by

$$
\begin{aligned}
& i_{\exp 1}(t)=k_{p}\left[y_{E 1}(t)-y_{B 1}(t)-z_{0}\right]+k_{d} \dot{y}_{E 1}(t)+i_{0}, \\
& i_{\exp 2}(t)=k_{p}\left[y_{E 2}(t)-y_{B 2}(t)-z_{0}\right]+k_{d} \dot{y}_{E 2}(t)+i_{0} .
\end{aligned}
$$

Here, $i_{\exp }(t)$ is the desired current flowing through the electromagnet and $k_{p}$ and $k_{d}$ are the coefficients of the proportionality and damping term of the controller, respectively. To accelerate the response of the actuators, a cascaded current controller with feed-forward is used $[15,18]$. Consider

$$
\begin{aligned}
& u_{1}(t)=k_{c}\left[i_{\exp 1}(t)-i_{1}(t)\right]+2 R i_{1}(t), \\
& u_{2}(t)=k_{c}\left[i_{\exp 2}(t)-i_{2}(t)\right]+2 R i_{2}(t) .
\end{aligned}
$$

The dynamic model of the interaction system with a module, air-springs and cabin is developed.

2.2. The Modeling of Bridge. Generally, the elevated bridge of the maglev route is simply supported, and the span is greatly larger compared with other dimensions, and the vertical deflection of the bridges when the self-excited vibration occurs is small compared with its span. Generally, the elevated bridge is modeled as a Bernoulli-Euler beam [2], which will be adopted in this paper. Based on the above assumptions, the motion of bridge can be described by the following differential equation

$$
E I_{b} \frac{\partial^{4} y(x, t)}{\partial x^{4}}+\rho_{b} \frac{\partial^{2} y(x, t)}{\partial t^{2}}=f(x, t),
$$

where $E I_{b}$ is the bending stiffness, $\rho_{b}$ is the mass per unit length, and $f(x, t)$ is the electromagnetic force acting on the bridge. For a simply supported bridge, the $k$ th natural frequency $\omega_{k}$ and mode shape $\phi_{k}(x)$ are

$$
\begin{gathered}
\omega_{k}=\lambda_{k}^{2} \sqrt{\frac{E I_{b}}{\rho_{b}}}, \\
\phi_{k}(x)=\sin \left(\lambda_{k} x\right) .
\end{gathered}
$$

Here, $\lambda_{k}=k \pi / L$. Using modal analysis method, the solutions of (15) can be expressed by the linear superposition of modal shapes. Consider

$$
y(x, t)=\sum_{k=1}^{\infty} \phi_{k}(x) q_{k}(t)
$$

Substituting (4) into (1), multiplying both sides of the resultant equation by $\sin (n \pi x / L)$, and integrating both sides from 0 to $L$, then

$$
\ddot{q}_{k}(t)+\omega_{k}^{2} q_{k}(t)=\frac{2}{\rho_{b} L} \int_{0}^{L} f(x, t) \phi_{k}(x) d x .
$$


Considering that the length of the electromagnet is approximately $1 / 20$ of bridge, the electromagnetic force may be viewed as a concentrated force, taking into account the modal damping of bridge; then,

$$
\ddot{q}_{k}(t)+2 \xi_{k} \omega_{k} \dot{q}_{k}(t)+\omega_{k}^{2} q_{k}(t)=\frac{2}{\rho_{b} L} \sum_{i=1}^{n} \phi_{k}\left(x_{c i}\right) F_{E i}(t) .
$$

Multiplying both sides of the resultant equation by $\phi_{k}\left(x_{c}\right)$ gives

$$
\ddot{y}_{k}(t)+2 \xi_{k} \omega_{k} \dot{y}_{k}(t)+\omega_{k}^{2} y_{k}(t)=\frac{2}{\rho_{b} L} \sum_{i=1}^{n} \phi_{k}^{2}\left(x_{c i}\right) F_{E i}(t) .
$$

Here, $y_{k}(t)$ is the $k$ th modal displacement of the bridge.

2.3. The Modeling Comparison of the Levitation Systems. It has been observed that the self-excited vibration occurs when the vehicle is suspended upon the guideway, standing still or moving at very slow speed [4]. When the speed of vehicle is larger than $10 \mathrm{~km} / \mathrm{s}$ approximately, during the period of the vehicle passing over a beam, the energy accumulated by the bridge is limited, and the vibration amplitude of the bridge is sufficiently small which cannot be observed. Hence, in the present study, the vehicle is assumed to be standing still without moving. In this situation, the crossing of the vehicle between the joints of the beams is not considered.

When the vibration amplitude of the bridge is sufficiently small, the interaction system is quasistatic, so that the linearized model may be applied to explore the principle underlying the self-excited vibration without introducing noticeable errors. The linearized equations with a module, the air-springs, and cabin in frequency domain are

$$
\begin{gathered}
a_{11} y_{E 1}(s)+a_{12} y_{E 2}(s)+a_{15} i_{1}(s)=b_{1} s q_{1}(s), \\
a_{12} y_{E 1}(s)+a_{11} y_{E 2}(s)+a_{15} i_{2}(s)=b_{2} s q_{1}(s), \\
a_{31} y_{E 1}(s)+a_{32} y_{E 2}(s)+a_{35} i_{1}(s)+a_{37} F_{E 1}(s)=b_{3} s q_{1}(s), \\
a_{32} y_{E 1}(s)+a_{31} y_{E 2}(s)+a_{35} i_{2}(s)+a_{37} F_{E 2}(s)=b_{4} s q_{1}(s), \\
a_{51} y_{E 1}(s)+a_{52} y_{E 2}(s)+a_{53} y_{C 1}(s)+a_{54} y_{C 2}(s) \\
+a_{57} F_{E 1}(s)+a_{58} F_{E 2}(s)=0, \\
a_{52} y_{E 1}(s)+a_{51} y_{E 2}(s)+a_{54} y_{C 1}(s) \\
+a_{53} y_{C 2}(s)+a_{58} F_{E 1}(s)+a_{57} F_{E 2}(s)=0, \\
a_{71} y_{E 1}(s)+a_{72} y_{E 2}(s)+a_{73} y_{C 1}(s)+a_{74} y_{C 2}(s)=0, \\
a_{72} y_{E 1}(s)+a_{71} y_{E 2}(s)+a_{74} y_{C 1}(s)+a_{73} y_{C 2}(s)=0,
\end{gathered}
$$

where $a_{11}=\left[k_{p} k_{c} s+\left(k_{d} k_{c}+1.5 F_{i}\right) s^{2}\right], a_{12}=0.5 F_{i} s^{2}$, $a_{15}=-\left(k_{c} s+2 L_{0} s^{2}\right), a_{31}=1.5 F_{z} s, a_{32}=0.5 F_{z} s, a_{35}=-2 F_{i} s$, $a_{37}=s, a_{51}=\left(k_{s} \eta_{3}+c_{s} \eta_{3} s-s^{2}\right), a_{52}=\left(k_{s} \eta_{4}+c_{s} \eta_{4} s\right)$, $a_{53}=-\left(k_{s} \eta_{3}+c_{s} \eta_{3} s\right), a_{54}=-\left(k_{s} \eta_{4}+c_{s} \eta_{4} s\right), a_{57}=\eta_{1}$, $a_{58}=\eta_{2}, a_{71}=\left(k_{s} \sigma_{1}+c_{s} \sigma_{1} s\right), a_{72}=\left(k_{s} \sigma_{2}+c_{s} \sigma_{2} s\right)$, $a_{73}=-\left(k_{s} \sigma_{1}+c_{s} \sigma_{1} s+s^{2}\right), a_{74}=-\left(k_{s} \sigma_{2}+c_{s} \sigma_{2} s\right)$, $b_{1}=\left[k_{p} k_{c} \sin \left(\lambda_{1} x_{B 1}\right)+2 F_{i} \sin \left(\lambda_{1} x_{B 1}+\lambda_{1} L_{E}\right) s\right], b_{2}=$ $\left[k_{p} k_{c} \sin \left(\lambda_{1} x_{B 2}\right)+2 F_{i} \sin \left(\lambda_{1} x_{B 2}-\lambda_{1} L_{E}\right) s\right], \quad b_{3}=$ $2 F_{z} \sin \left(\lambda_{1} x_{B 1}+\lambda_{1} L_{E}\right)$, and $b_{4}=2 F_{z} \sin \left(\lambda_{1} x_{B 2}-\lambda_{1} L_{E}\right)$. According to (21), there are nine variables in eight equations. Here, $s q_{1}(s)$ is defined as an input, and $\left[\begin{array}{llllllll}y_{E 1}(s) & y_{E 2}(s) & y_{C 1}(s) & y_{C 2}(s) & i_{1}(s) & i_{2}(s) & F_{E 1}(s) & F_{E 2}(s)\end{array}\right]^{T}$ is defined as the states of the interaction system. Then, the transfer functions $T_{1}(s)=F_{E 1}(s) / s q_{1}(s)$ and $T_{2}(s)=$ $F_{E 2}(s) / s q_{1}(s)$ can be solved. Furthermore, the averaged work in a period $P_{1}=\sin \left(\lambda_{1} x_{B 1}+\lambda_{1} L_{E}\right) / T \cdot \int_{0}^{T} F_{E 1}(t) v_{1}(t) d t$ and $P_{2}=\sin \left(\lambda_{1} x_{B 2}-\lambda_{1} L_{E}\right) / T \cdot \int_{0}^{T} F_{E 2}(t) v_{1}(t) d t$ can be obtained (Figure 5).

This is to say, half of the averaged work $P_{M}=0.5\left(P_{1}+P_{2}\right)$ acting on the bridge will be gotten. Similarly, the interaction system with a single levitation unit and its work $P_{S}$ acting on the bridge may be solved. Considering the limited length of the paper, the derivation process is omitted here.

Assuming that the self-excited vibration occurs and the vibration frequency $\omega_{\mathrm{Vib}}$ is less than the crossing frequency $\omega_{c}$, the work acting on the bridge done by the levitation system is negative, which means that the levitation system absorbs the vibration energy. Together with the energy dissipated by the modal damping of bridge, the vibration energy of bridge will be consumed, which leads the amplitude of the vibration decay to zero.

According to the analysis, we can conclude that the vibration frequency of vibration will be greater than $\omega_{c}$. This is to say, as long as the work acting on the bridge done by the levitation is negative, no matter how large the averaged quantity of work is, the stability of the interaction system will be held in the range of $\left[\begin{array}{ll}0 & \omega_{c}\end{array}\right]$. Hence, the concavedown curve of $P_{M}$ at $1.6 \mathrm{~Hz}$ (which is the natural vibration frequency of air springs) does not affect the stability of the interaction system.

On condition in which the vibration frequency $\omega_{\mathrm{Vib}}$ is greater than the critical frequency $\omega_{c}$, the work acting on the bridge done by the levitation system will be positive, which may lead to the growth of the vibration. Therefore, we should pay more attention to the frequency range $\left[\begin{array}{ll}\omega_{c} & \infty\end{array}\right]$.

The active character of the levitation system is the essence of the self-excited vibration when the frequency is greater than $\omega_{c}$. In light of the comparison between the powers $P_{M}$ and $P_{S}$, a high coherence is kept when the frequency is greater than $\omega_{c}$.

Comprehensively, we can conclude that the interaction model with a single levitation unit and a simply supported Bernoulli-Euler beam is the minimum model for the study of the self-excited vibration.

\section{The Influences of Parameters on the Stability of the Interaction System}

It has been observed that the PD controller cannot stabilize the vehicle-bridge interaction system completely on different bridges. However, yet as of today, to the authors' knowledge, 


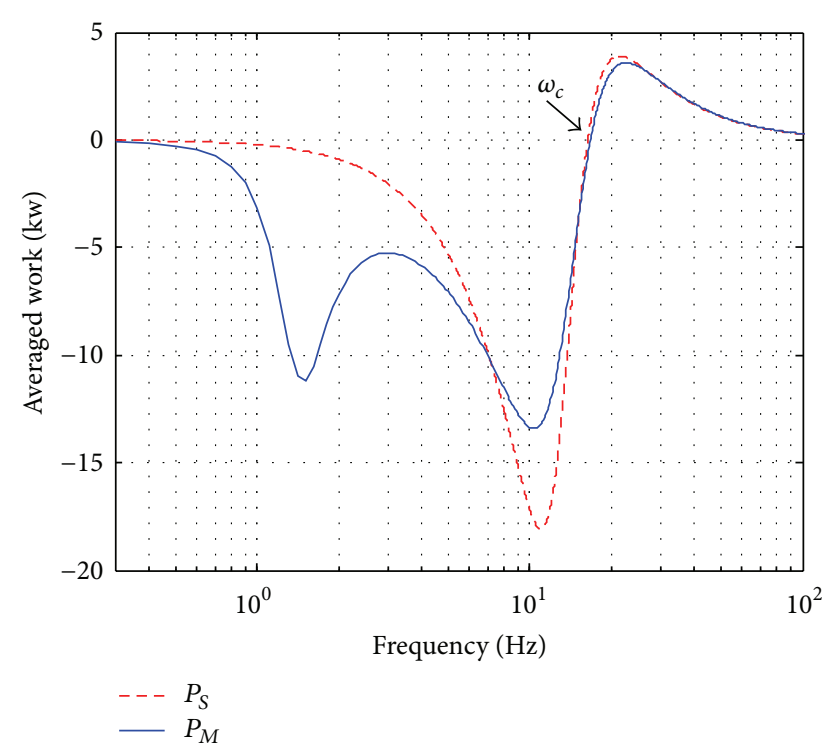

Figure 5: The comparison of the works done by different levitation models.

few literatures about the influences of the system parameters on the stability of the self-excited vibration based on the minimum interaction model have been published.

To study the influences, the dynamic equations of the minimum interaction system with a levitation unit and a simply supported Bernoulli-Euler beam in frequency domain are

$$
\begin{gathered}
-2 F_{z} y_{1}(s)+2 F_{z} y_{0}(s)+2 F_{i} i(s)-F_{m}(s)=0, \\
m_{1} \sigma s^{2} y_{1}(s)+\left(s^{2}+2 \xi \omega s+\omega^{2}\right) y_{0}(s)=0, \\
\left(k_{p} k_{c}+2 F_{i} s+k_{d} k_{c} s\right) y_{1}(s)-k_{p} k_{c} \delta_{0}(s) \\
-\left(k_{p} k_{c}+2 F_{i} s\right) y_{0}(s)-\left(k_{c}+2 L_{0} s\right) i(s)=0 .
\end{gathered}
$$

It has been observed that the bridge vibrates vertically when the self-excited vibration occurs. With the passage of time, the amplitude of vibration increases until the failure of the levitation system or self-oscillation with constant amplitude. To some extent, the fluctuation of the levitation gap is the main marker of self-excited vibration. Therefore, the transfer function between the levitation gap $\delta(t)$ and desired levitation gap $\delta_{0}(t)$ is creditable to judge the stability of the interaction system.

Eliminating $i(s)$ and $F_{m}(s)$ of (22), the transfer function between $\delta(s)$ and $\delta_{0}(s)$ is

$$
G_{0}(s)=\frac{\delta(s)}{\delta_{0}(s)}=-\frac{b_{1} s^{2}}{a_{5} s^{5}+a_{4} s^{4}+a_{3} s^{3}+a_{2} s^{2}+a_{1} s+a_{0}} .
$$

In this section, $b_{1}=2 m_{1} k_{p} k_{c} F_{i} \sigma, a_{5}=2 L_{0} m_{1}, a_{4}=$ $m_{1}\left(4 \xi \omega L_{0}+k_{c}\right), a_{3}=2 k_{d} k_{c} F_{i}+2 k_{c} \xi \omega m_{1}+2 L_{0} m_{1} \omega^{2}, a_{2}=$ $2 k_{p} k_{c} F_{i}+k_{c} m_{1} \omega^{2}+4 k_{d} k_{c} F_{i} \xi \omega+2 \sigma m_{1} k_{c} k_{p} F_{i}-2 \sigma m_{1} k_{c} F_{z}-$ $2 k_{c} F_{z}$, and $a_{1}=-4 \xi \omega k_{c} F_{z}+2 k_{d} k_{c} F_{i} \omega^{2}$. According to the characteristic roots of $G_{0}(s)$, the stability analysis of the interaction system near the equilibrium point is solved.

Furthermore, in maglev engineering, mastering the tendency of the stability of the interaction system when the parameters are varying is vital to stabilize the physical system. The root locus method may be used to study the effects of the varying parameters on closed-loop pole locations. For the root locus method, only a varying parameter per analysis should be adopted, so that a cluster of nominal parameters should be determined in advance. Here, the parameters from the maglev engineering practice are listed:

the parameters of controllers: $k_{p}=5500, k_{d}=50$, and $k_{c}=30$,

the parameters of bridges: $\xi=0.01, \omega=62.8$, and $\rho_{b}=2000$,

the parameters of vehicle: $M_{1}=1150, m_{1}=500$.

3.1. The Influences of the Parameters of Bridge. For the bridges, the damping ratio $\xi$ and modal frequency $\omega$ are related to the stability of the vehicle-bridge interaction system. The characteristic equation of the transfer function (23) may be written as

$$
\begin{aligned}
& 2 L_{0} m_{1} s^{5}+m_{1}\left(4 \xi \omega L_{0}+k_{c}\right) s^{4} \\
& +\left(2 k_{d} k_{c} F_{i}+2 k_{c} \xi \omega m_{1}+2 L_{0} m_{1} \omega^{2}\right) s^{3} \\
& +\left(2 k_{p} k_{c} F_{i}+4 k_{d} k_{c} F_{i} \xi \omega+k_{c} m_{1} \omega^{2}+2 \sigma m_{1} k_{c} k_{p} F_{i}\right. \\
& \left.\quad-2 \sigma m_{1} k_{c} F_{z}-2 k_{c} F_{z}\right) s^{2} \\
& +\left(4 \xi \omega k_{p} k_{c} F_{i}+2 k_{d} k_{c} F_{i} \omega^{2}-4 \xi \omega k_{c} F_{z}\right) s \\
& +\omega^{2}\left(2 k_{p} k_{c} F_{i}-2 k_{c} F_{z}\right)=0 .
\end{aligned}
$$

Considering that the damping ratio $\xi$ can be separated from the characteristic equation (24), to plot the root locus diagram by the command rlocus of MATLAB software, it should be translated into the standard form as follows:

$$
\begin{gathered}
d(s)+\xi \cdot n(s)=0 \\
d(s)=2 L_{0} m_{1} s^{5}+k_{c} m_{1} s^{4}+\left(2 k_{d} k_{c} F_{i}+2 L_{0} m_{1} \omega^{2}\right) s^{3} \\
+\left(2 k_{p} k_{c} F_{i}+k_{c} m_{1} \omega^{2}+2 \sigma m_{1} k_{c} k_{p} F_{i}\right. \\
\left.-2 \sigma m_{1} k_{c} F_{z}-2 k_{c} F_{z}\right) s^{2} \\
+2 k_{d} k_{c} F_{i} \omega^{2} s+2 k_{p} k_{c} F_{i} \omega^{2}-2 k_{c} F_{z} \omega^{2} \\
n(s)=4 \omega L_{0} m_{1} s^{4}+2 k_{c} \omega m_{1} s^{3} \\
+4 k_{d} k_{c} F_{i} \omega s^{2}+\left(4 \omega k_{p} k_{c} F_{i}-4 \omega k_{c} F_{z}\right) s .
\end{gathered}
$$

Then, the root locus diagram about the varying damping ratio of the bridges with different modal frequencies, $5 \mathrm{~Hz}$ and $20 \mathrm{~Hz}$, is shown in Figure 6.

According to Figure 6, when the damping ratio is less than 1.0 , the greater the damping ratio is, the better the 


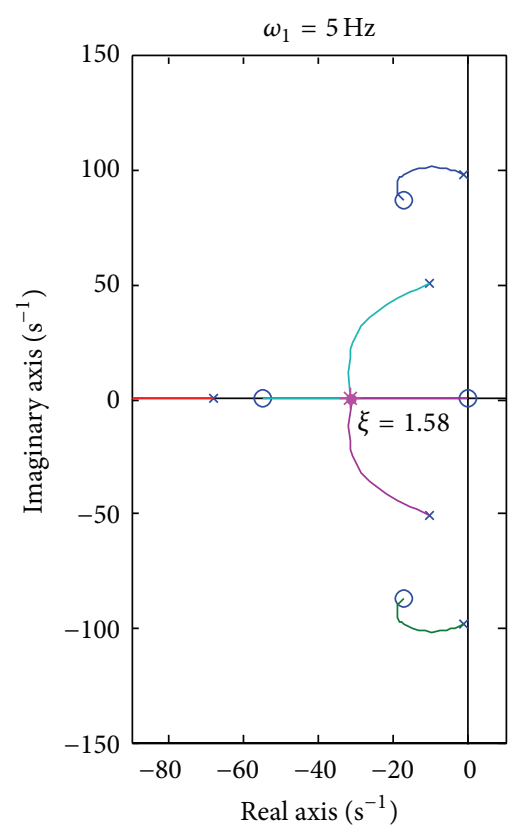

(a)

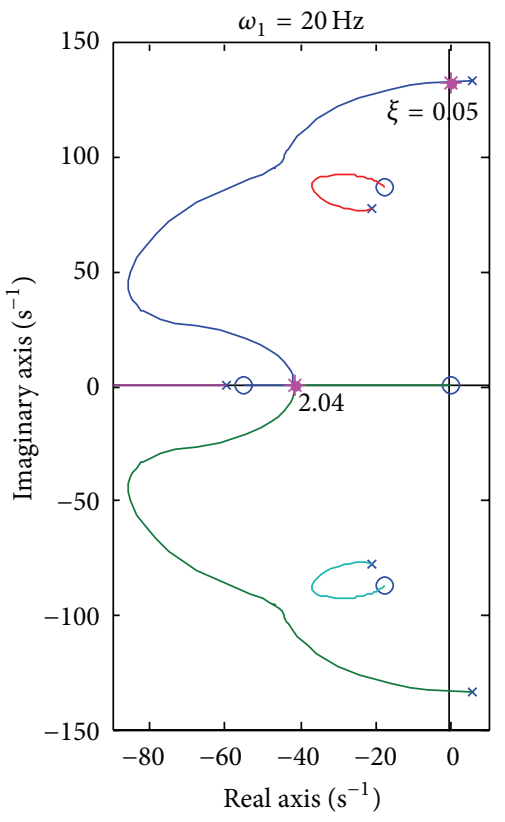

(b)

FIgURE 6: The root locus about the varying damping ratios.

stability of the interaction system will be. However, the damping ratio of the bridge is mainly determined by the character of its material and is almost independent of its span and mass per unit length. According to the field records from the maglev engineering test, the damping ratio is about 0.01 , and the variation between different bridges is tiny.

Furthermore, for the bridges, whose first modal frequency is $5 \mathrm{~Hz}$, the interaction system is stable no matter how large the damping ratio is. However, for the bridge, whose modal frequency is $20 \mathrm{~Hz}$, the self-excited vibration may appear if the damping ratio is less than 0.05 . Therefore, the stability of the interaction system is closely related with the modal frequency of the bridges.

Due to the existence of both linear term $\omega$ and quadratic term $\omega^{2}$, the terms associated with the modal frequency are hard to be separated from the characteristic equation (24). This is to say, the characteristic equation cannot be translated into the standard form. On this condition, the root locus diagram about the varying modal frequency $\omega$ should be plotted manually, which is shown in Figure 7.

According to Figure 7, the interaction system is unstable if the modal frequency $\omega$ is greater than $57 \mathrm{rads}^{-1}$ and less than $230 \mathrm{rads}^{-1}$. Considering that the first modal frequency of the bridges in Tangshan maglev engineering base is less than $62.8 \mathrm{rads}^{-1}$ mostly, we can conclude that the lower modal frequency of bridge is beneficial to the stability of the interaction system.

In maglev field, a consensus has been reached that the heavier the bridge is, the better the stability of the interaction system will be. However, litter reasonable theoretical analysis has been reported. The root locus diagram about the mass per unit length $\rho_{b}$ is shown in Figure 8. There are five root

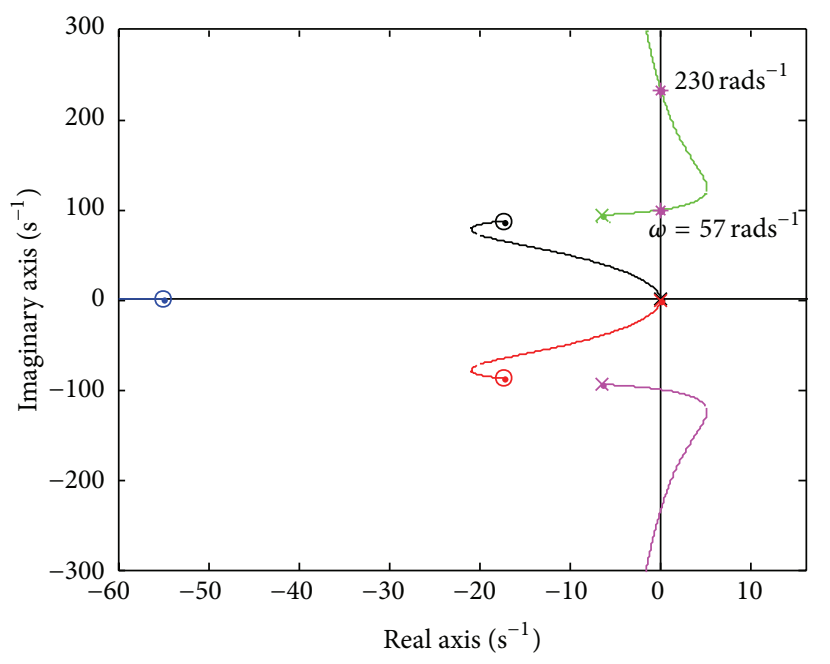

FIGURE 7: The root locus about the varying first modal frequencies.

contours, and only one contour is unstable when $\rho_{b}$ is less than $862 \mathrm{kgm}^{-1}$.

3.2. The Influences of the Parameters of PD Controller. For the maglev system, if a control scheme can stabilize the vehiclebridge interaction system for any kind of bridges, it may be perfect. However, for the aforementioned PD controller, it is incompetent unless the optimal parameters are selected. To obtain the optimal parameters, the influences of each parameter on the stability of the interaction system should be studied extensively. 


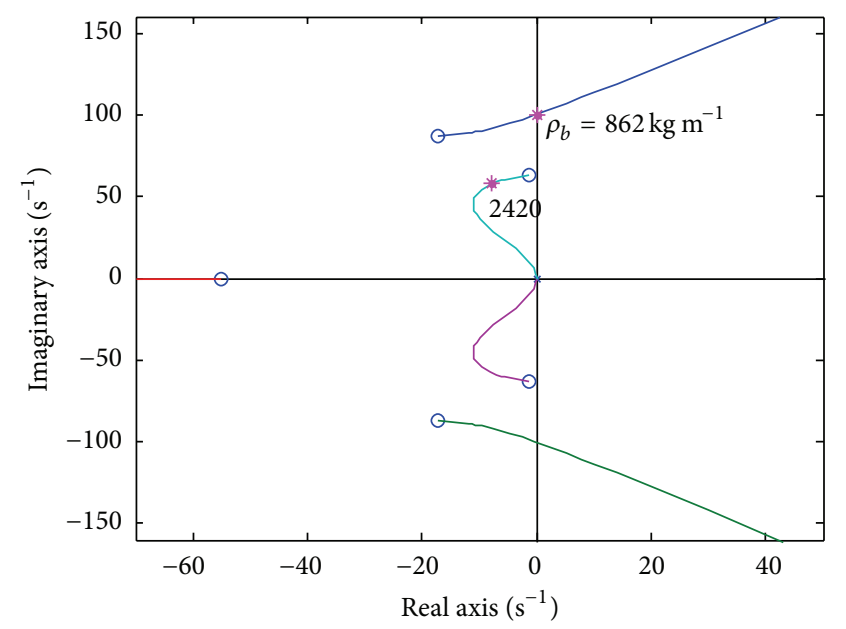

FIgURE 8: The root locus about the varying mass per unit lengths.

First of all, the influence of the parameter $k_{p}$ is shown in Figure 9. There are five root contours, whose stabilities are all related with the variation of $k_{p}$. Due to the symmetry along real axis, the analysis may be simplified to the upper three contours. For the first contour, the parameter $k_{p}$ should be less than 6280 . For the second contour, the parameter $k_{p}$ should be greater than 3700 . For the third contour, the parameter $k_{p}$ should be greater than 3830 . Comprehensively, the parameter $k_{p}$ should be less than 6280 and greater than 3830 in the normal case of others parameters.

For the integral-type PD controller, the velocity signal is from the integration of the acceleration of the electromagnet. The influences of the $k_{d}$ are shown in Figure 10. Comprehensively, $k_{d}$ should be greater than 47.8 . Besides, to some extent, we can conclude that the greater the parameters $k_{d}$ is, the better the stability of the interaction system will be.

The inner loop (current feedback) accelerates the response of the electromagnets. Generally, the greater the parameter $k_{c}$ is, the faster the response of the actuator will be. According to Figure 11, we can conclude that the $k_{c}$ should be greater than 24.1. Besides, to some extent, the greater the parameters $k_{c}$ is, the better the stability of the interaction system will be.

3.3. The Influences of the Sprung and Unsprung Mass. The maglev vehicle is divided into two sections by air springs, an unsprung section and a sprung section. Generally speaking, the mass of unsprung section is reviewed as a constant value. In spite of the unsprung mass being invariable after the completion of its construction, the analysis about the unsprung mass on the stability may be significant for the design of the unsprung section, which is shown in Figure 12.

For the CMS04 maglev system, the mass of unsprung section is about $500 \mathrm{~kg}$, which is less than the critical value $961 \mathrm{~kg}$. To a certain extent, the influence of the variation of the unsprung mass on the stability of the interaction system is insignificant.

The mass of the sprung section is uncertain due to the addition of luggage and passengers. For a levitation unit,

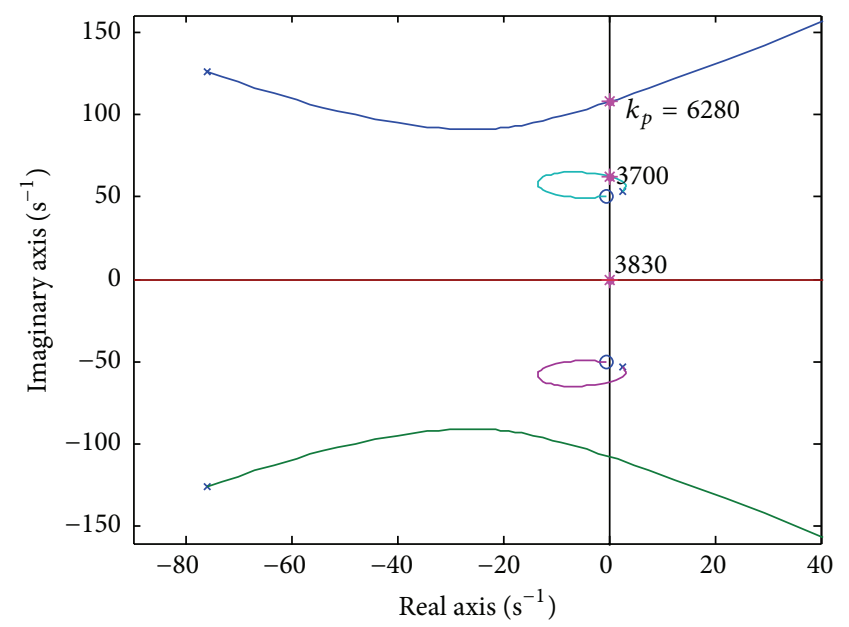

FIgURE 9: The root locus diagram about the varying $k_{p}$.

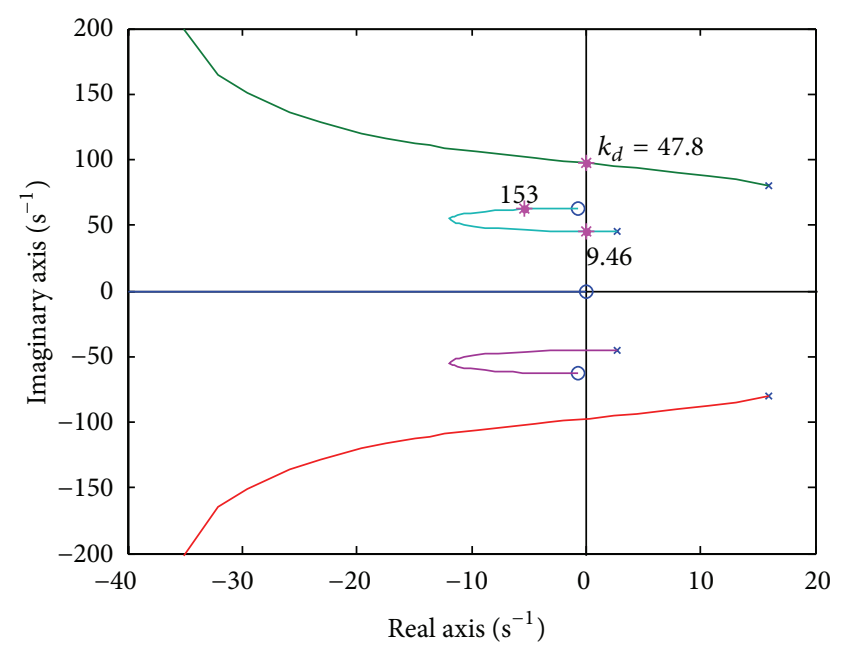

FIGURE 10: The root locus diagram about the varying $k_{d}$.

its sprung mass varies between $650 \mathrm{~kg}$ and $1250 \mathrm{~kg}$. The influence of the variation of sprung mass on the stability of the interaction system is shown in Figure 13. Comprehensively, the sprung mass should be greater than $1041 \mathrm{~kg}$ and less than $4140 \mathrm{~kg}$. In light of the full load of sprung mass being less than $1250 \mathrm{~kg}$, in the case of normal parameters, the stability of the interaction system with the full load is better than the interaction system with no load.

\section{Conclusions}

After the investigation on the modeling and analysis of the influences on the stability of the interaction system, we can draw the following conclusions. Firstly, an index that reflects the essence of the self-excited vibration is defined, and a minimum interaction model containing a levitation unit and a Bernoulli-Euler beam is developed. Secondly, the principle underlying the self-excited vibration is explored. The active character of the levitation above the critical frequency $\omega_{c}$ is the root. Thirdly, the influences of the parameters, including 


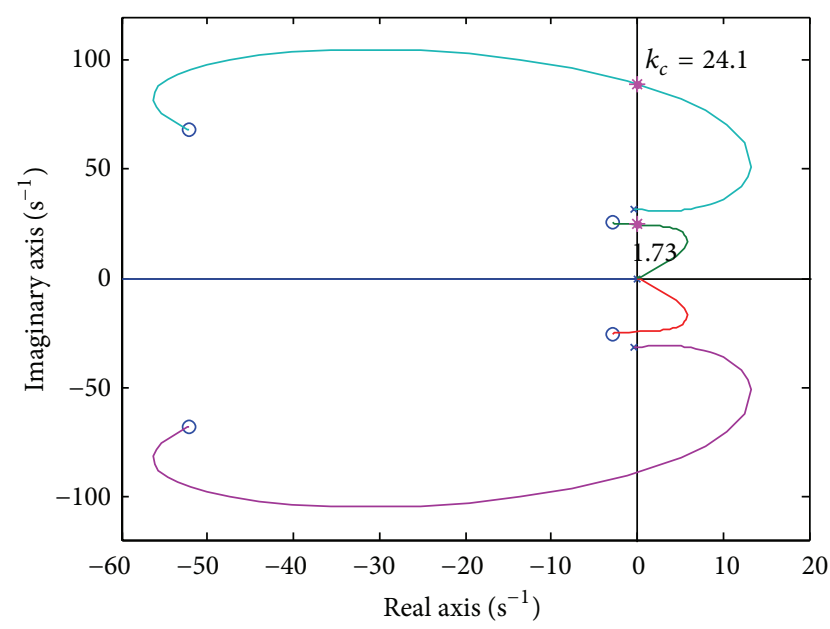

FIGURE 11: The root locus diagram about the varying $k_{c}$.

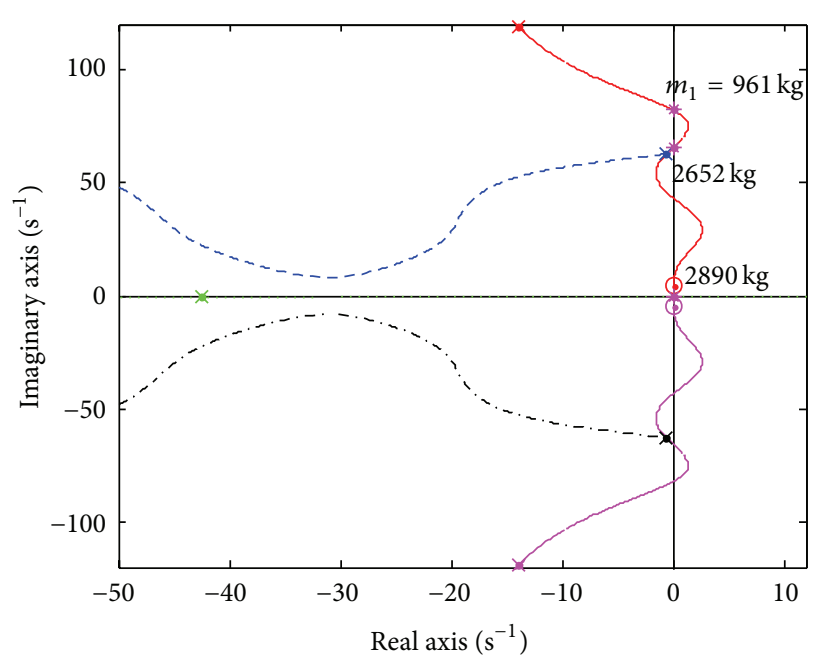

FIGURE 12: The root locus about the varying unsprung masses.

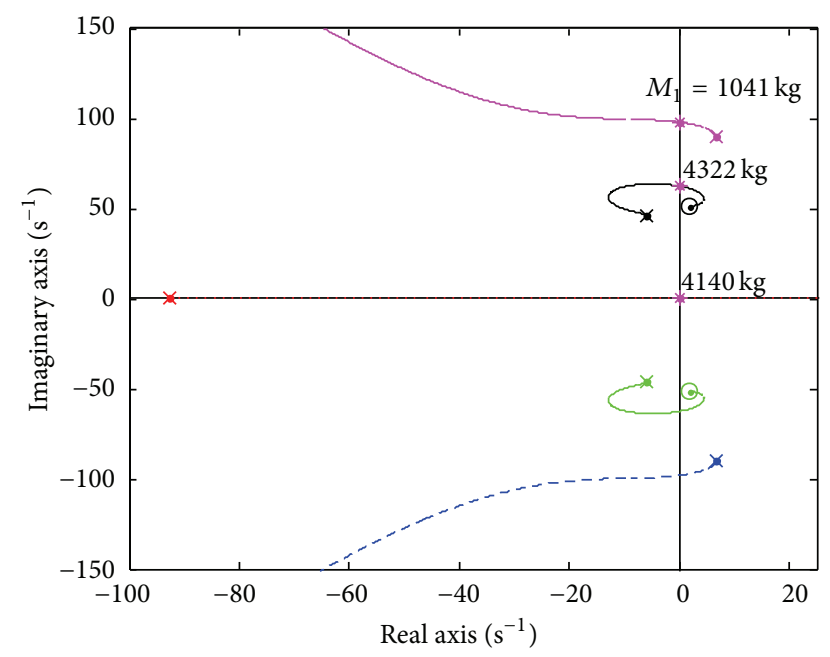

FIGURE 13: The root locus about the varying sprung masses. the modal frequency and the damping ratio of the bridge, the parameters of the controller, the sprung mass, and unsprung mass of vehicle are analyzed according to the roots locus method. To some extent, the lower modal frequency, the larger damping ratio and mass per unit length, and the higher gain of $k_{d}$ and $k_{c}$ are beneficial to the stability of the interaction system, which provides a theoretical guidance for solving the self-excited vibration problems.

\section{Conflict of Interests}

The authors declare that there is no conflict of interests regarding the publication of this paper.

\section{Acknowledgment}

The authors gratefully acknowledge the financial support supported by the National Natural Science Foundation of China (nos. 11202230 and 11302252).

\section{References}

[1] N. G. Elvin and A. A. Elvin, "An experimentally validated electromagnetic energy harvester," Journal of Sound and Vibration, vol. 330, no. 10, pp. 2314-2324, 2011.

[2] D. F. Zhou, C. H. Hansen, and J. Li, "Suppression of maglev vehicle-girder self-excited vibration using a virtual tuned mass damper," Journal of Sound and Vibration, vol. 330, no. 5, pp. 883901, 2011.

[3] R.-J. Wai, J.-D. Lee, and K.-L. Chuang, "Real-time PID control strategy for maglev transportation system via particle swarm optimization," IEEE Transactions on Industrial Electronics, vol. 58, no. 2, pp. 629-646, 2011.

[4] D. Zhou, C. H. Hansen, J. Li, and W. Chang, "Review of coupled vibration problems in EMS maglev vehicles," The International Journal of Acoustics and Vibrations, vol. 15, no. 1, pp. 10-23, 2010.

[5] T. E. Alberts, G. Oleszczuk, and A. M. Hanasoge, "Stable levitation control of magnetically suspended vehicles with structural flexibility," in Proceedings of the American Control Conference (ACC '08), pp. 4035-4040, Seattle, Wash, USA, June 2008.

[6] D.-S. Zou, L.-H. She, Z.-Q. Zhang, and F.-M. Zhou, "Maglev vehicle and guideway coupling vibration analysis," Acta Electronica Sinica, vol. 38, no. 9, pp. 2071-2075, 2010.

[7] L. Li and G. Meng, "The analysis of coupling vibration between maglev vehicle and steel bridge," Journal of Vibration and Shock, vol. 25, no. 6, pp. 46-75, 2006.

[8] H. P. Wang, J. Li, and K. Zhang, "Vibration analysis of the maglev guideway with the moving load," Journal of Sound and Vibration, vol. 305, no. 4-5, pp. 621-640, 2007.

[9] H. P. Wang, J. Li, and K. Zhang, "Sup-resonant response of a nonautonomous maglev system with delayed acceleration feedback control," IEEE Transactions on Magnetics, vol. 44, no. 10, pp. 2338-2350, 2008.

[10] X. Li, L. Zhang, Z. Zhang, L. She, and L. Huang, "Bifurcation control for maglev system with two state feedback delays," in Proceedings of the IEEE International Conference on Information and Automation (ICIA '08), pp. 244-247, June 2008.

[11] Z. Z. Zhang and L. L. Zhang, "Hopf bifurcation of time-delayed feedback control for maglev system with flexible guideway," 
Applied Mathematics and Computation, vol. 219, no. 11, pp. 61066112, 2013.

[12] H.-S. Han, B.-Y. Yim, N.-J. Lee, Y.-C. Hur, and J.-G. Kwon, "Vibration analysis of a maglev vehicle using electromagnetic suspension," in Proceedings of the International Conference on Electrical Machines and Systems (ICEMS '07), pp. 1963-1969, Seoul, Republic of Korea, October 2007.

[13] Y.-G. Li, D. Zhang, and H. Cheng, "The design and simulation of an adaptive maglev control algorithm based on oscillation observation," in Proceedings of the 18th International Conference on Magnetically Levitated Systems and Linear Drives (MAGLEV '04), pp. 984-990, Shanghai, China, October 2004.

[14] E. Kong, J.-S. Song, B.-B. Kang, and S. Na, "Dynamic response and robust control of coupled maglev vehicle and guideway system," Journal of Sound and Vibration, vol. 330, no. 25, pp. 6237-6253, 2011.

[15] Y.-G. Li and W.-S. Chang, "Cascade control of an EMS maglev vehicle's levitation control system," Acta Automatica Sinica, vol. 25, no. 2, pp. 247-251, 1999.

[16] D.-F. Zhou and J. Li, "Analysis of the low-frequency vibration of EMS maglev vehicle," in Proceedings of the IEEE International Conference on Control and Automation, pp. 3157-3161, Guangzhou, China, June 2007.

[17] Y. Tian and L. H. She, "Stability analysis of maglev elastic vehicle-guideway-coupled system," in Proceedings of the IEEE International Conference on Computer Science and Automation Engineering, pp. 215-219, Shanghai, China, June 2011.

[18] V. M. H. Guzmán and R. S. Ortigoza, "Current loops in a magnetic levitation system," International Journal of Innovative Computing, Information and Control, vol. 5, no. 5, pp. 1275-1283, 2009. 


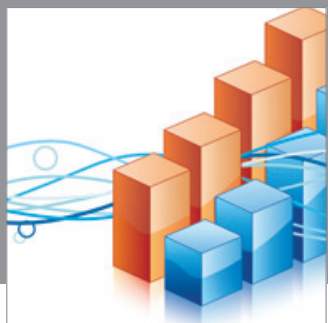

Advances in

Operations Research

mansans

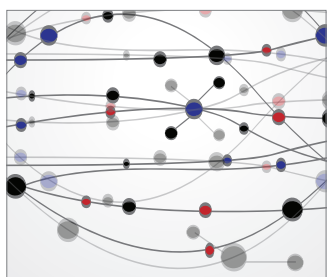

The Scientific World Journal
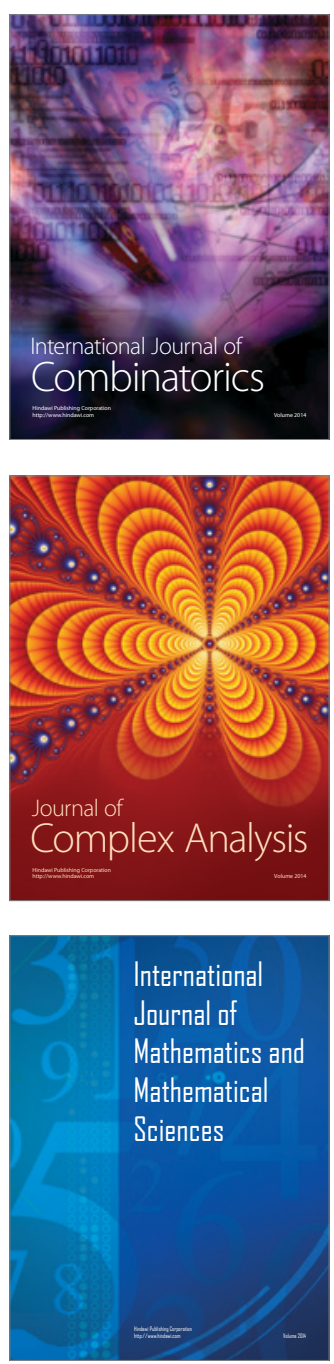
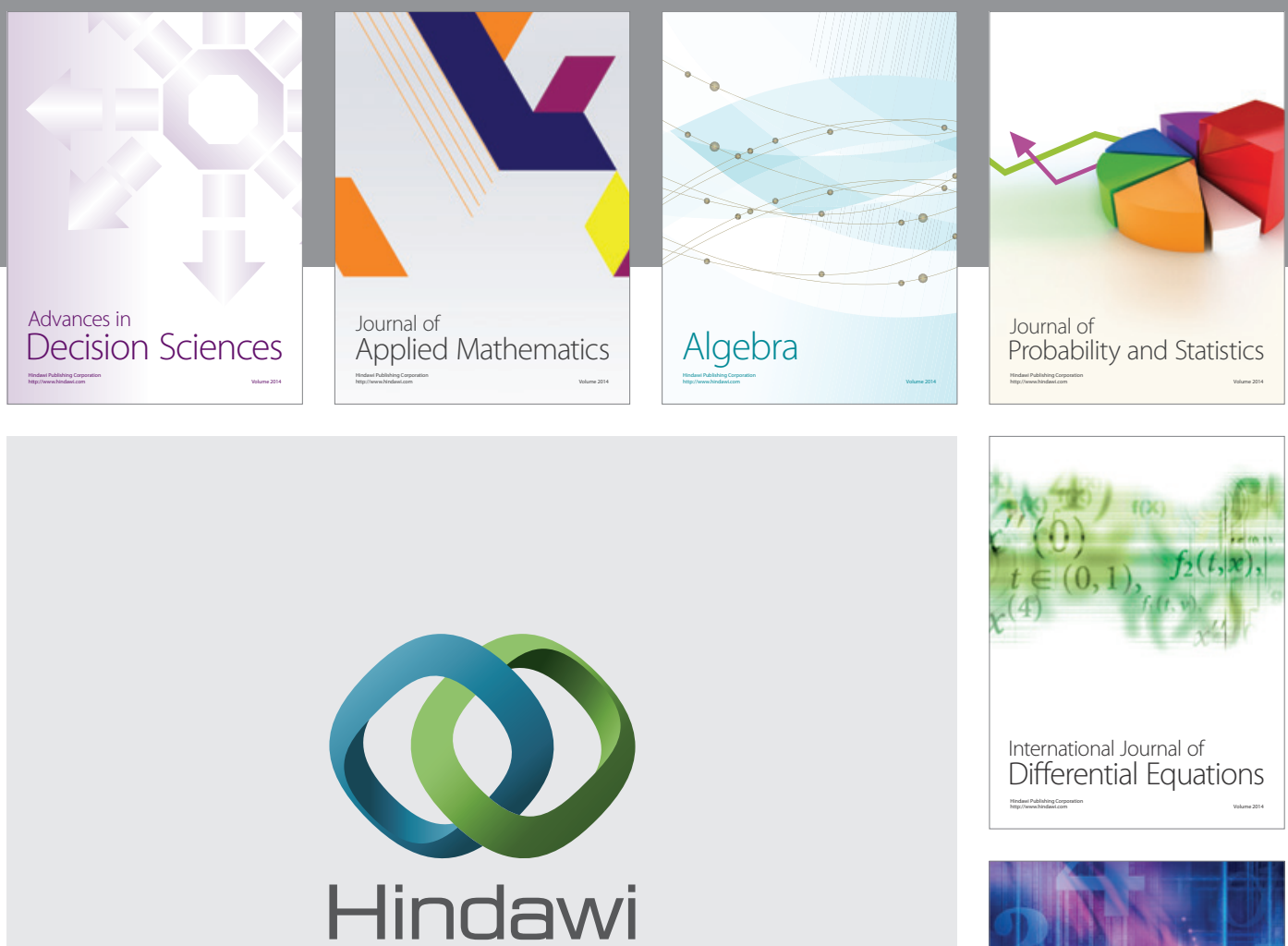

Submit your manuscripts at http://www.hindawi.com
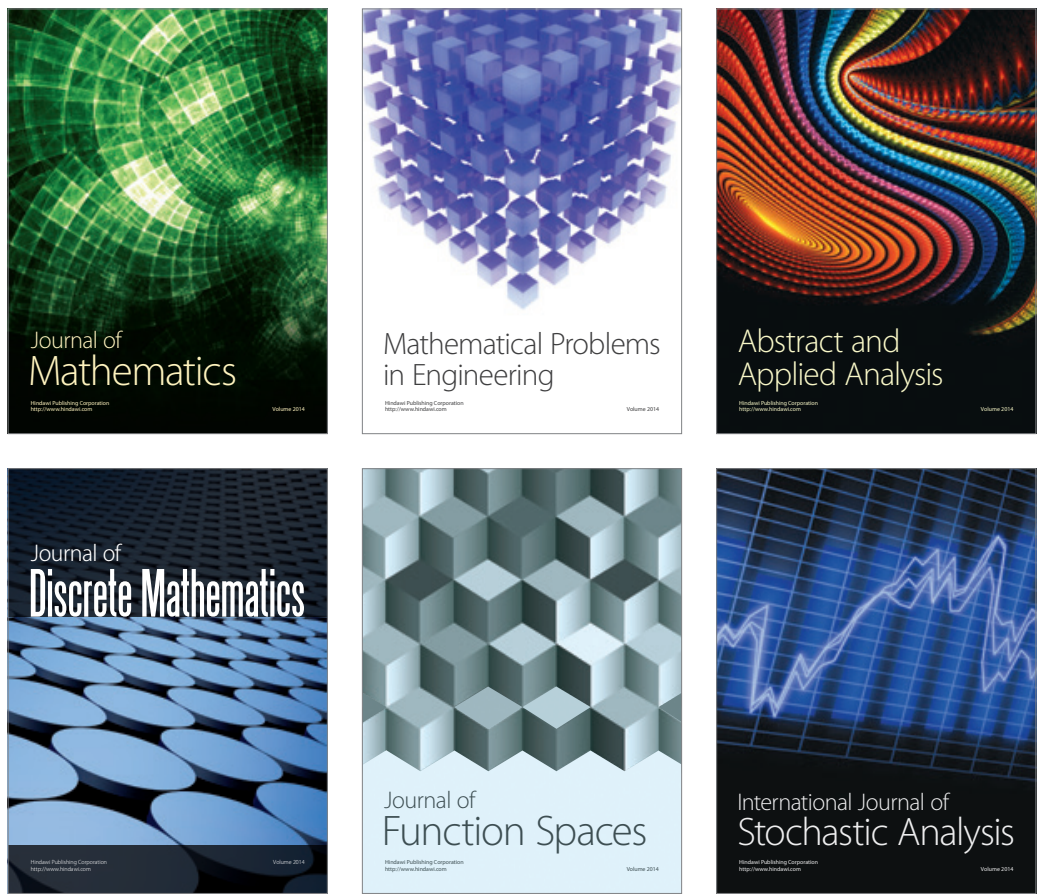

Journal of

Function Spaces

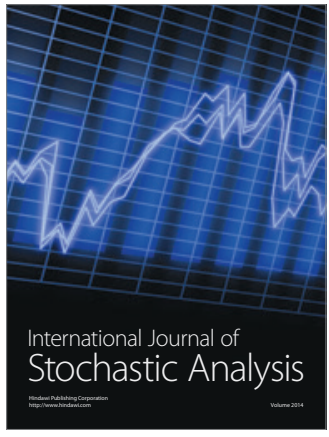

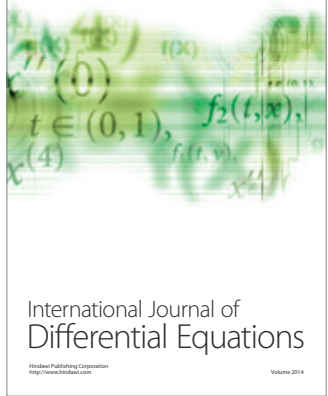
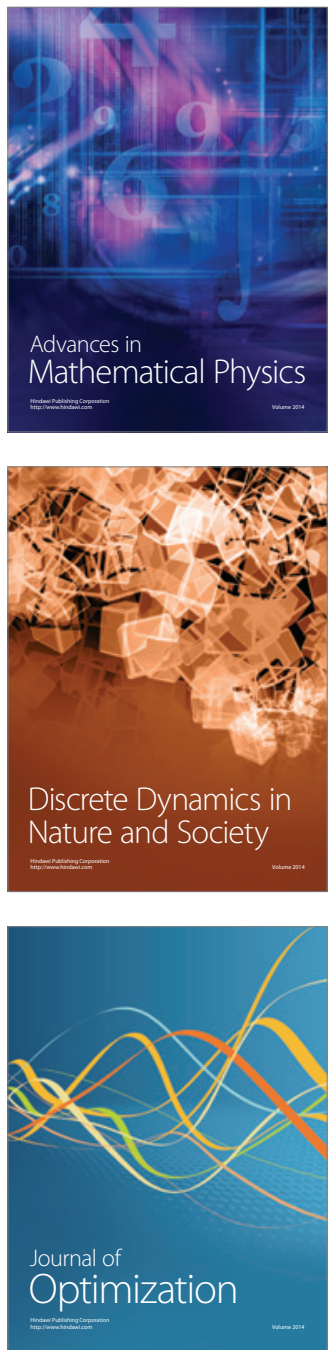